\title{
Simultaneous Phase Shifting Shearing Interferometry for Measurement of Static and Dynamic Phase Objects
}

\author{
Noel-Ivan Toto-Arellano스, David-Ignacio Serrano-García ${ }^{1}$, \\ Amalia Martínez-García ${ }^{1}$ and Gustavo Rodríguez-Zurita ${ }^{2}$ \\ ${ }^{1}$ Centro de Investigaciones en Óptica A.C. León, Gto., \\ ${ }^{2}$ Benemérita Universidad Autónoma de Puebla, \\ México
}

\section{Introduction}

Through the use of non-destructive optical techniques to measure the surface with high accuracy, two shearing interferometers were implemented to analyze the slope of phase objects using simultaneous phase-shifting shearing interferometry [Toto et al., 2010]. These optical configurations allow obtaining $n$-shearograms simultaneously to retrieve the optical phase data map with higher accuracy [Rodriguez et al., 2009; Toto et al 2009].

Several optical systems have been developed to retrieve the optical phase data in a single capture employing polarization, for example, through the use of micro-polarizer arrays [Novak et al.,2005], a point diffraction interferometer[Neal, 2003 ], a two-beam grating interferometer [Rodriguez, 2008] and a liquid-crystal spatial modulator [Mercer and Creath, 1996], among others. These systems have been employed in several fields of application, such as optical metrology[Wyant, 2004; Cheng and Wyant, 1984], holography[Yamaguchi and Zhang, 1997; Nomura, 2006], optical tomography[Meneses, 2006], ESPI [Chen, 2010], etc. In this chapter, we present theoretical analyses and experimental results obtained by implementing several shearing simultaneous phaseshifting interferometers capable of retrieving the directional derivatives in the $x$ direction, or adapting the systems to obtain radial derivatives [Serrano et al., 2011], allowing direct slope measurements; in each case, the optical phase data are processed by means of the capture of $n$-interferograms in a single shot. To achieve this, we propose an alternative to the interferometers developed for phase shifting that use micro polarizer arrays, replacing them with a $4-f$ system with high frequency Ronchi gratings or Phase gratings coupled at the end of the interferometer.

In order to obtain the partial derivative of the phase object, a Cyclic Shear Interferometer (CSI) of lateral shear with crossed circular polarization has been implemented; the CSI is coupled to a $4-f$ system with high frequency Ronchi gratings, a pair of crossed gratings $G(\mu, v)$ placed as the system's pupil with a spatial period $d$. The high frequency Bi-Ronchi gratings generate replicas of the patterns required to process the derivative of the phase. The 
processing of the interferograms is performed using conventional methods of phase extraction with the algorithm of four shifts. For the case when the object presents radial symmetry, it is more convenient to use a radial-shear interferometer. Applications of radialshear interferometers have been previously reported in optical testing [ Malacara, 1974; Steel 1965], aspherical surface measuring [Hariharan, 1984], corneal topographic inspection [Kowalik et al., 2002], adaptive optics [Shirai et al., 2001], wavefront sensing [Geary, 2000; Hutchin 1985] and beam characterization [Hernandez, 2000; Liu, 2007], among others, for these cases, we propose the use of a Mach-Zehnder Radial-Shear Interferometer (MZRI), consisting of one telescope system on each arm.

The advantage of both systems is its ability to obtain the derivative of the object wavefront in real time, which can be associated with the partial or radial slope of the phase object, as will be seen in the following sections; additionally, the phase gratings used in these systems can be replaced by high frequency Bi-Ronchi gratings. Experimental results are presented for phase objects.

\section{Lateral and radial shearing interferometers}

Obtaining the derivative of the wavefront brings numerous advantages due to the high sensitivity against abrupt phase changes. In some cases, if the derivatives in the $x$ and $y$ directions are known, we can calculate the gradient information of the object, but this implies the design of optical systems that generate shearing in both directions, or we can obtain shearing in each direction separately. If the object under study presents radial symmetry, it is more convenient to use a radial-shear interferometer. In this section, we present in detail two interferometric systems used to generate directional derivatives and the slope associated, using simultaneous phase shifting interferometry.

\subsection{Cyclic path interferometer}

Figure 1(a) shows the experimental set-up to generate the derivative of the incident wave front in the $x$-direction. It consists of a cyclic path interferometer where the illumination comes from a polarized Verdi laser underneath, operating at $\lambda=532 \mathrm{~nm}$. The system is illuminated with polarized light at $45^{\circ}$ with respect to the $x$-axis (using a quarter-wave retarding plate, $\mathrm{Q}$, and a linear polarizer, $\mathrm{P})$.

The PBS generates two beams with crossed linear polarization states, which become circular after going through a wave retardation plate of $\lambda / 4$ (Q'). Thus, the output of the CSI consists of two sheared wavefronts, each one with mutually orthogonal circular polarizations(Left and Right). The interference pattern generated has circular crossed polarizations, and the shear can be varied by moving mirror $M^{\prime}$. At the output of the CSI, a $4-f$ system has been implemented, with two crossed high frequency Ronchi gratings placed on the Fourier plane [Toto et al., 2010](The Ronchi gratings can be substituted by Phase grids, taking into account the proper considerations at the moment of selecting the interference replicas to calculate the phase data map). For this case, shear $\Delta x=x_{0}$ has a smaller value than size a of the beam's cross section, which is smaller than order separation $F_{0}$ ( Two neighboring diffraction orders have a distance of $F_{0} \equiv \lambda f / d$ on the image plane for a grating); thus, two beams with shear $\Delta x$ enter the $4-f$ system close to the optical axes. At the image plane, the 
superposition of the two beam replicas with mutual shear $\Delta x$ would appear around each diffraction order. Since the interferograms retain their polarization characteristics, polarizing filters are placed in each generated replica in order to generate the phase shifts required to obtain the phase. Fig. 1(b) shows the two configurations of the polarizing arrangement proposed to generate the phase shifts in a single shot, used to process the optical phase. Some replicas of the interference patterns have different intensities; however, there are at least four patterns with similar intensities in each configuration. As will be shown in the following sections, the replicas of the pattern generated by the CSI are centered on each diffraction order [Rodriguez, 2009].

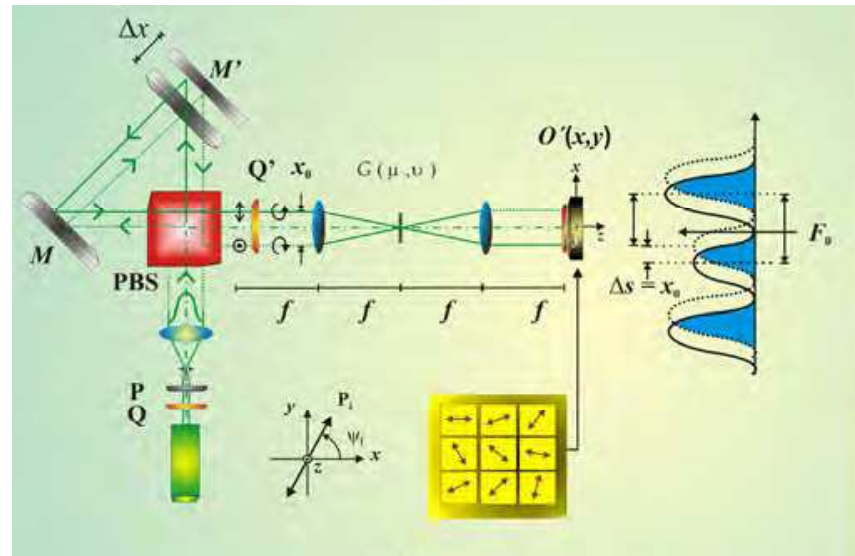

(a)

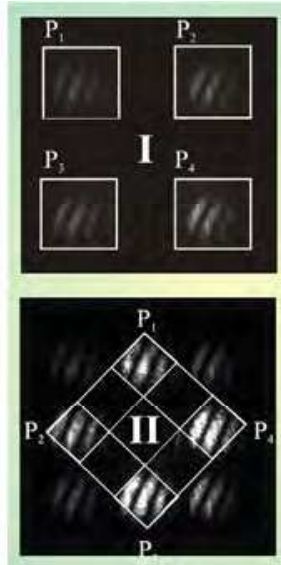

(b)

Fig. 1. Simultaneous phase shifting cyclic path interferometer. (a) CSI with variable shear coupled to $4-f$ system with high frequency Bi-Ronchi grating. $Q, Q$ ': Quarter wave retarders. $\Delta x$ : linear shear. $x_{0}$ : beam separation. $F_{0}=\lambda f / d$ is the order of separation. (b) Arrangement of polarizing filters. $P_{\mathrm{i}}$ : polarizing filters, $\Psi_{\mathrm{i}}$ : transmission angle of polarization.

\subsection{Mach Zehnder interferometer}

In several cases, such as in ophthalmic lens analysis, radial symmetry is encountered, and it is strongly necessary to obtain the radial derivative of the incident wave front in order to analyze the radial variations of the objects. For that reason, we propose the use of another optical system to obtain the radial derivative and associate it with its corresponding radial slope. Fig. 2 shows the experimental setup for the measurement of the radial slope of transparent objects. The interferometric system uses a He-Ne laser operating at $\lambda=632.8 \mathrm{~nm}$. Polarized light at $45^{\circ}$ enters the interferometer by using a quarter-wave retarder plate $Q_{0}$ and a linear polarizer $\mathrm{P}_{0}$. The Mach-Zehnder radial-shear interferometer (MZRI) presented comprises two telescope systems, (S1, S2) [Lago and de la Fuente R. ,2008] one on each arm with vertical and horizontal linear polarized light, respectively. Their variants are widely used as part of different applications, so they can be considered to be representative cases of potential adaptations for simultaneous phase shifting interferometry. A $4-f$ system is coupled at the end of the interferometer; this system consists of two similar achromatic 
lenses of focal length $f \approx 20 \mathrm{~cm}$ and a phase grid $\mathrm{G}(\mu, \mathrm{v})$ placed as the system's pupil with spatial period $d=110 \mathrm{ln} / \mathrm{mm}$. In the phase grid used, $\mu=u / \lambda f$ and $v=v / \lambda f$ are the frequency coordinates $(u, v)$ scaled to wavelength $\lambda$ and focal length $f$. The output of the MZRI consists of two versions of the same wavefront, but slightly enlarged in relation to the copy, and each one with mutually orthogonal linear polarizations (vertical and horizontal) too. A wave retarder plate of $\lambda / 4\left(Q_{1}\right)$ is used to obtain cross circular polarization for each radial-shear wavefront (left and right, $\vec{J}_{L}$ and $\vec{J}_{R}$ ) with equal amplitudes[Toto et al., 2009].

A linear polarized filter array is placed on the image plane, where each one is centered on its respective replicas of the interference pattern, as it is shown in Fig. 2 of the polarized linear array. The cross sections of the two sheared beams are $a_{1}=7.0 \mathrm{~mm}$ and $a_{2}=8.6 \mathrm{~mm}$, and the relative magnification of the pupils is $M_{a}=1.23$.

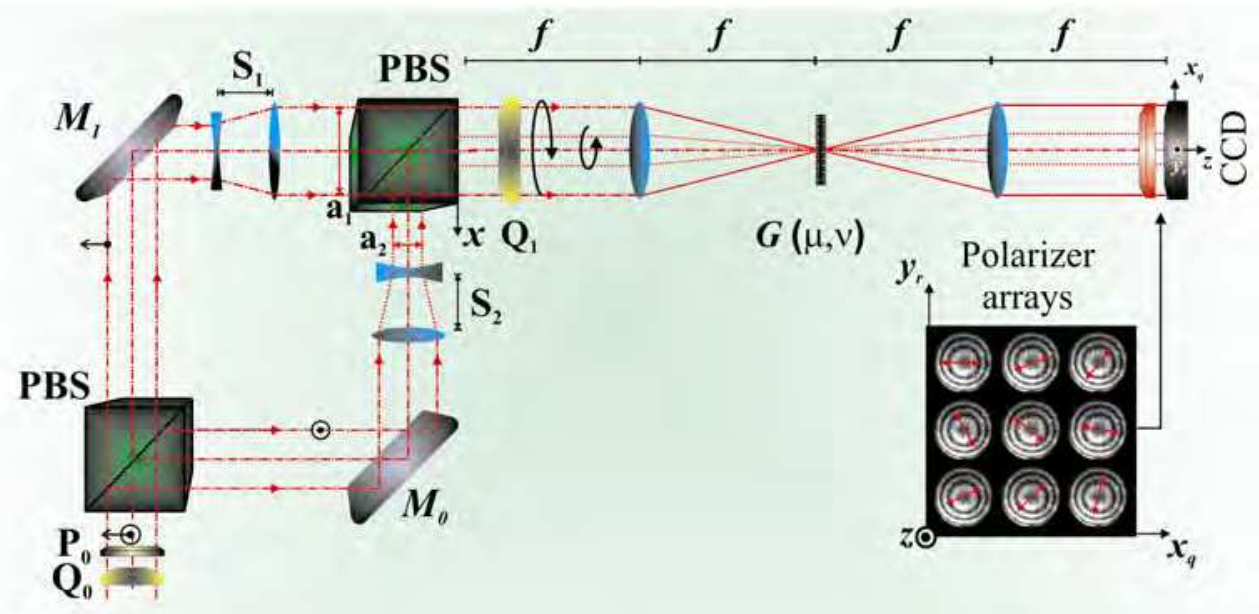

Fig. 2. Simultaneous phase shifting Mach-Zehnder radial-shear interferometer. $P_{\mathrm{i}}$ : Polarizers; $Q_{\mathrm{i}}$ : QWP operating at $632.8 \mathrm{~nm}$; PBS: Polarizing Beam splitter; $M_{\mathrm{i}}$ : Mirrors; $\mathrm{G}(\mu, \mathrm{v})$ : Phase grid. Translation of coordinates around the order position: $x_{q}=x-q F_{0}$ and $y_{r}=y-r F_{0}$.

In the present section, we analyze the general case of the interference patterns obtained by the shearing interferometers presented, as well as the modulation by polarization generated in each replica in both cases (lateral or radial shear) by the Jones's Matrix representation.

\section{Polarizing phase-shifting lateral shear interferometry}

At the output of the CSI, two images are generated, each with circular polarization; the two fields generated for the system are described by

$$
O(x+\Delta x, y)=\frac{1}{\sqrt{2}}\left(\begin{array}{l}
1 \\
i
\end{array}\right) e^{i \phi(x+\Delta x, y)} \quad O(x, y)=\frac{1}{\sqrt{2}}\left(\begin{array}{c}
1 \\
-i
\end{array}\right) e^{i \phi(x, y)}
$$


Eq. (1) represents cross circular polarization states of same beams with lateral shear $\Delta x$. When each field is observed through a linear polarizing filter whose transmission axis is at an angle $\psi$, the new polarization states are

$$
O^{\prime}(x, y)=P_{\psi} \cdot O(x+\Delta x, y), O^{\prime \prime}(x, y)=P_{\psi} \cdot O(x, y)
$$

where $P_{\psi}$ is the matrix for a linear polarizer with its axis of transmission at angle $\psi$, given by

$$
P_{\psi}=\left(\begin{array}{cc}
\cos ^{2} \psi & \sin \psi \cos \psi \\
\sin \psi \cos \psi & \sin ^{2} \psi
\end{array}\right) .
$$

When the two fields interfere, the resulting irradiance can be written as

$$
\begin{aligned}
& I(x, y)=\left\|O^{\prime}(x, y)+O^{\prime \prime}(x, y)\right\|^{2} \\
& I(x, y)_{i}=2+2 \cos \left\{2 \psi_{i}-[\phi(x+\Delta x, y)-\phi(x, y)]\right\} . \\
& I(x, y)_{i}=2 \cdot\left\{1+\cos \left[2 \psi_{i}-\Delta x \frac{\partial}{\partial x} \phi(x, y)\right]\right\}
\end{aligned}
$$

When $i=1 \ldots 4$, the relative phase can be calculated as [Sharma, 1984; Barrientos, 1999]

$$
\frac{\partial}{\partial x} \phi(x, y)=\tan ^{-1}\left(\frac{I_{1}-I_{3}}{I_{2}-I_{4}}\right),
$$

where $I_{i}$ are the intensity measurements captured in a single shot, with the values of $\psi$ given by $\psi_{1}=0 \quad \psi_{2}=46.577^{\circ} \quad \psi_{3}=92.989^{\circ} \quad \psi_{4}=136.42^{\circ}$. Hence, the slope is:

$$
\frac{\partial w(x, y)}{\partial x}=\frac{1}{c_{1}} \cdot \tan ^{-1}\left(\frac{I_{1}-I_{3}}{I_{2}-I_{4}}\right),
$$

with $c_{1}=\lambda / 4 \pi \cdot \Delta x$.

\section{Polarizing phase-shifting radial shear interferometry}

Assuming each beam section at the end of the MZRI as $O(r)=\operatorname{circ}[r] \cdot \exp \{i \phi(r)\}$ and $O(r+\Delta r)=\operatorname{circ}[r+\Delta r] \cdot \exp \{i \phi(r+\Delta r)\}$, the field entering the $4-f$ system is given by

$$
O_{1}(r)=\left(\begin{array}{l}
1 \\
i
\end{array}\right) \cdot O(r)+\left(\begin{array}{c}
1 \\
-i
\end{array}\right) \cdot O(r+\Delta r)
$$

representing cross circular polarization states of the same beam radially sheared. When each field is observed through a linear polarizing filter whose transmission axis is at angle $\psi$, the new polarization states are: 


$$
O_{2}(r)=\cdot P_{\psi}\left[\left(\begin{array}{l}
1 \\
i
\end{array}\right) \cdot O(r)+\left(\begin{array}{c}
1 \\
-i
\end{array}\right) \cdot O(r+\Delta r)\right] \text {. }
$$

As it was shown in section 3, we can show that the interference pattern can be represented as:

$$
I(r)_{i}=2 \cdot\left\{1+\cos \left[2 \psi_{i}-\Delta r \frac{\partial}{\partial r} \phi(r)\right]\right.
$$

and considering also that $i=1 \ldots 4$, the relative phase data map can be calculated as

$$
\frac{\partial}{\partial r} \phi(r)=\tan ^{-1}\left(\frac{I_{1}-I_{3}}{I_{2}-I_{4}}\right),
$$

where $I_{i}$ are the intensity measurements captured in a single shot. In this particular case, the radial slope can be obtained as:

$$
\frac{\partial w(r)}{\partial r}=\frac{1}{c_{1}} \cdot \tan ^{-1}\left(\frac{I_{1}-I_{3}}{I_{2}-I_{4}}\right)
$$

\section{Grating interferometry: pattern replication and modulation of polarization}

\subsection{Interferometer with Bi-Ronchi gratings}

In the one-dimensional case, with $\mu$ denoting the object space, the amplitude transmission of a Ronchi grating [Ronchi ,1964.] can be written as the periodic function

$$
G(\mu)=\operatorname{rect}\left[\frac{\mu}{a_{w}}\right] * \sum_{n=-N}^{N} \delta\left[\frac{\mu-n \cdot d}{a_{w}}\right],
$$

with $d=a_{w}+b_{w}$ being the grating period, $a_{\mathrm{w}}$ and $b_{w}$ being the widths of the clear and dark bands respectively, $\delta(\mu)$ denoting the Dirac delta function, and * the convolution. $\mu=u / \lambda f$ is the frequency coordinate scaled to the relevant wavelength $\lambda$ and the focal length $f$. The Fourier transform of Eq. (12) with $N \rightarrow \infty$ is given by

$$
\begin{aligned}
\tilde{G}(x) & =\frac{a_{w}}{d} \operatorname{sinc}\left(a_{w} x\right) \sum_{n=-\infty}^{\infty} \delta\left(x-\frac{n}{d}\right) \\
& =\frac{a_{w}}{d} \sum_{n=-\infty}^{\infty} \operatorname{sinc}\left(a_{w} \frac{n}{d}\right) \delta\left(x-\frac{n}{d}\right)=\sum_{n=-\infty}^{\infty} C_{n} \cdot \delta\left(x-\frac{n}{d}\right)
\end{aligned}
$$

where $C_{n}$ results as the $n$-Fourier complex coefficient of $G(\mu)$. In general, when Bi-Ronchi gratings[Cordero, 1998] are placed on the Fourier plane, they can be considered to be two cross amplitude Ronchi gratings, 


$$
G(\mu, v)=G(\mu) \cdot G(v)=\sum_{n=-N}^{N} \operatorname{rect}\left[\frac{\mu-n \cdot d_{\mu}}{a_{w \mu}}\right] \cdot \sum_{l=-L}^{L} \operatorname{rect}\left[\frac{v-l \cdot d_{v}}{a_{w \mu}}\right]
$$

were $N, L$ are the numbers of components of the grating, $d_{\mu}, d_{v}$ are the respective periods along directions " $\mu$ " and " $v$ ", and $a_{w_{\mu}}, a_{w_{v}}$ are the widths in clear strips along each one of both directions. The gratings studied have equal periods in both directions; then, $d_{\mu}=d_{v}=d$, as well as $a_{w \mu}=a_{w v}=a_{w}$. Due to the properties of the Fourier transform, the corresponding spectrum is:

$$
\begin{aligned}
\tilde{G}(x, y)= & \tilde{G}(x) \cdot \tilde{G}(y) \\
& =\frac{a_{w}{ }^{2}}{d^{2}} \sum_{n=-N}^{N} \operatorname{sinc}\left(\frac{a_{w}}{d} \cdot x\right) \cdot \delta\left(x-\frac{n}{d}\right) \sum_{l=-L}^{L} \operatorname{sinc}\left(\frac{a_{w}}{d} y\right) \cdot \delta\left(y-\frac{l}{d}\right) \\
& =\sum_{n=-N}^{N} C_{x n} \cdot \delta\left(x-\frac{n}{d}\right) \sum_{l=-L}^{L} C_{y l} \cdot \delta\left(y-\frac{l}{d}\right)
\end{aligned}
$$

For the experimental case where gratings $(100 \mathrm{ln} / \mathrm{mm})$ have the same period and $d=0.01 \mathrm{~mm}$, the diffraction patterns generated using Eq. (15) are shown in Fig. 3. Fig. 3(a) shows the simulated diffraction pattern generated by the Bi-Ronchi grating. In Fig. 3(b), it can be seen that the amplitude spectra for each axis show more than three orders of diffraction; however, only orders 0 and \pm 1 allow one to obtain replicas of the interference pattern with comparable intensities, the fringe modulation it is close to unity.

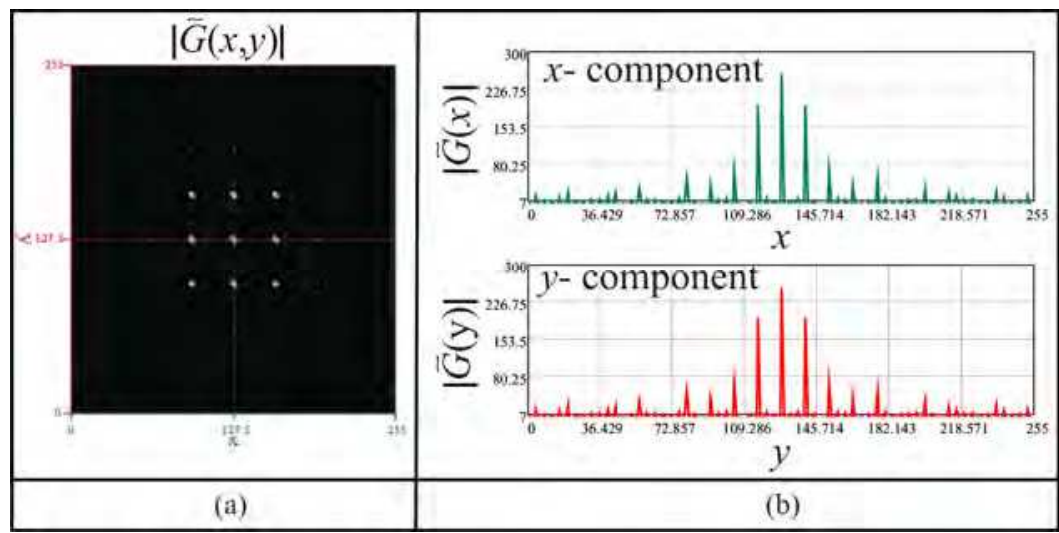

Fig. 3. Simulated diffraction pattern generated by high frequency Bi-Ronchi grating. (a) Diffraction spectra. (B) $x-y$ components of diffraction spectra of each grating component.

\subsubsection{Interference pattern replication}

The 4- $f$ system with high frequency Bi-Ronchi grating only multiplexes the interference pattern generated at the output of CSI; the shifts in the interference pattern can be generated operating linear polarizers placed at an angle $\psi$. Considering Eq. (1), the interference pattern $I^{\prime}(x, y)$ on the image plane of the system will be: 


$$
\begin{aligned}
I^{\prime}(x, y) & =\left|\vec{P}_{\psi}\left[\vec{J}_{L} O(x+\Delta x, y)+\vec{J}_{R} O(x+, y)\right] * \widetilde{G}(x, y)\right|^{2}, \\
& =I(x, y) *|\widetilde{G}(x, y)|^{2},
\end{aligned}
$$

where each sheared wavefront has a circular polarization, $\vec{J}_{L}$ (left) and $\vec{J}_{R}$ (right) respectively, and $I(x, y)$, the interference pattern generated by the CSI, having the form

$$
I=1+\cos \left[2 \cdot \psi-\Delta x \frac{\partial}{\partial x} \phi(x, y)\right]
$$

so, the interference pattern on the image plane, centered on each diffraction order, will be:

$$
\begin{aligned}
& I^{\prime}(x, y)=\sum_{n=-N}^{N} \sum_{l=-L}^{L} C_{y l}^{2} \cdot C_{x n}^{2} \cdot I\left(x-\frac{n}{d_{x}}, y-\frac{l}{d_{y}}\right) \\
& =\sum_{n=-N}^{N} \sum_{l=-L}^{L} C_{y l}^{2} \cdot C_{x n}{ }^{2} \cdot\left\{1+\cos \left[2 \cdot \psi-\Delta x \frac{\partial}{\partial x} \phi\left(x-\frac{n}{d_{x}}, y-\frac{n}{d_{y}}\right)\right]\right\}
\end{aligned}
$$

Eq. (18) shows that the results are replicas of the pattern generated by the CSI displaced to the $n^{\text {th }}$ and $l^{\text {th }}$ orders of diffraction; each replica of the main pattern maintains an intensity modulated by Fourier Coefficients $C_{n}$ and $C_{l}$ corresponding to each direction. It should be noted that the contrast of the fringes depends on the degree of circular polarization of the interfering beams [Rodriguez, 2008]. In this case, the Ronchi gratings only multiplex the pattern, so the amplitudes of the orders only increase or decrease the intensity of the interference patterns, as it is shown in Fig. 4. Figure 4 shows the experimental results obtained with a Bi-Ronchi grating. The interference patterns used are shown enclosed in a circle, independent phase shifting, can be generated operating linear polarizers. Fig. 4(a) shows the diffraction orders' overlap, indicated by a subscript. Fig. 4(b) shows the diffraction orders generated by the grating; it can be seen that they have at least 9 diffraction orders with comparable intensities. This result is due to the modulation generated by coefficients $C_{n}$ and $C_{l}$ (see Eq. 18). Fig. 4(c) shows the replicas of the interference patterns centered on each diffraction order.

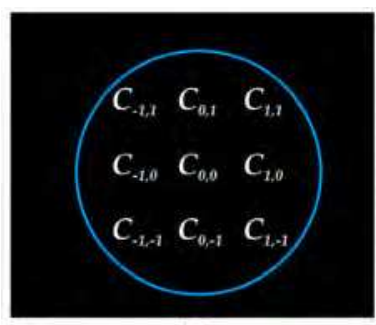

(a)

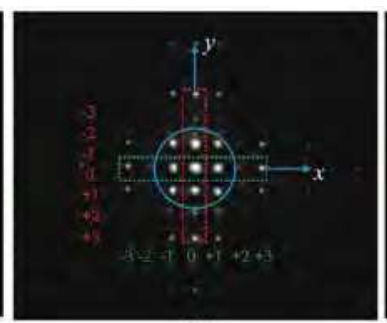

(b)

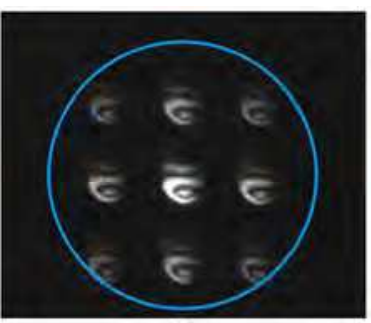

(c)

Fig. 4. Replicas of the interference patterns. (a) Fourier coefficients for each replica of the interference pattern. (b) Experimental diffraction orders generated by high frequency BiRonchi gratings (100 ln/mm). (c) Replicas of the interference patterns centered on each diffraction order (Circle). 


\subsection{Interferometer with phase grids}

Previous works have shown that when the interferometer is coupled to a $4-f$ system using phase grids, it is possible to obtain replicas of the interference pattern, and each one of these replicas can be modulated by polarization [Rodriguez 2008; Toto, 2008; Serrano, 2011]. A sinusoidal phase grid generated by the multiplication of two sinusoidal phase gratings, whose respective grating vectors are crossed, generates diffraction orders modulated by Bessel functions $J_{q}$ and $J_{r}$ [Rodriguez, 2008;Toto, 2008], and the image of the interference pattern generated by the interferometer is found centered around each of them. If we assume, for simplicity, that each beam section is of the form $w(x, y)=O(x) \cdot O(y) \cdot \exp \{i \phi(x, y)\}$, the transmittance in front of the entrance lens is given by

$$
\vec{t}_{i}(x, y)=\vec{J}_{L} \cdot w\left(x-\frac{\Delta x}{2}, y\right)+\vec{J}_{R} w\left(x+\frac{\Delta x}{2}, y\right)
$$

The displacement $\Delta x$ between the wavefronts can be adjusted by translating the mirror $M$ of the CSI. To draw theoretical conclusions, we assume that the phase grid is made up of two phase gratings (with $2 \Pi \mathrm{Ag}$ the grating's phase amplitude) and with orthogonal gratings vectors. Taking the rulings of one grating along the " $\mu$ " direction, and the rulings of the second grating along the " $v$ " direction, the resulting Fourier transform of the centered phase grid can be written as

$$
\tilde{G}_{2}(x, y)=\sum_{q=-\infty}^{q=\infty} \sum_{r=-\infty}^{r=\infty} J_{q}\left(2 \pi A_{g}\right) J_{r}\left(2 \pi A_{g}\right) \delta\left(x-q F_{0}, y-r F_{0}\right)
$$

where the order separation is $F_{0} \equiv \lambda f / d$, and $J_{q}, J_{r}$ denote the Bessel function of the first kind and integer order $q, r$ [Goodman, 1988]. On the image plane of the CSI, the amplitude can be written as

$$
\begin{aligned}
& \vec{t}_{o}(x, y)=\vec{t}_{i}(x, y) * \tilde{G}_{2}(x, y) \\
& =\sum_{q=-\infty}^{\infty} \sum_{r=-\infty}^{\infty} J_{q}\left(2 \pi A_{g}\right) J_{r}\left(2 \pi A_{g}\right)\left\{\vec{J}_{L} \cdot w\left(x-q F_{0}-\frac{\Delta x}{2}, y-r F_{0}\right)+\vec{J}_{R} \cdot w\left(x-q F_{0}+\frac{\Delta x}{2}, y-r F_{0}\right)\right\}
\end{aligned}
$$

with $(*)$ denoting convolution. On the image plane of the system, a series of replicated beams can be observed. Because of their polarization, an interference pattern can be detected when a linear polarizing filter is placed before the detector. Using Jones calculation with

$$
\vec{J}_{L}{ }^{\prime}=\mathbf{J}_{\psi}^{L} \vec{J}_{L}, \vec{J}_{R}{ }^{\prime}=\mathbf{J}_{\psi}^{L} \vec{J}_{R}, \quad \mathbf{J}_{\psi}^{L}=\left(\begin{array}{cc}
\cos ^{2} \psi & \sin \psi \cos \psi \\
\sin \psi \cos \psi & \sin ^{2} \psi
\end{array}\right),
$$

the pattern irradiance turns out to be proportional to the squared modulus of Eq. (21) in the general case. Considering the shear $\Delta x=x_{0}$ of a value smaller than the side $a$ of the beam section, which in turn is smaller than the order separation $F_{0}$ (Fig. 1), two beams with shear $x_{0}$ enter the $4-f$ system close to the optical axes. At the image plane, the superposition of the 
sheared beams would appear around each diffraction order, each superposition isolated from the others. The sheared beams around a given order $q r$ will have counter rotating circular polarization. A diffraction order $q r$ passes through a filter designed to block out all of the remaining orders. Under these conditions, by detecting the irradiance with a linear polarizing filter with the transmission axis at an angle $\psi$, only the contribution of an isolated term of order $q r$ can be considered, and its irradiance would be proportional to

$$
\begin{aligned}
& I(x, y)=\left(J_{q} J_{r}\right)^{2}+\left(J_{q} J_{r}\right)^{2}+2 J_{q} J_{q} J_{r} J_{r} \cdot \cos \left[\xi(\psi)-\Delta \phi\left(x_{q}, y_{r}\right)\right] \\
& \quad=2 J_{q}^{2} \cdot J_{r}^{2}\left(1+\cos \left[\xi(\psi)-\Delta \phi\left(x_{q}, y_{r}\right)\right]\right)
\end{aligned}
$$

where a translation of coordinates was used around the order position $\left(x_{q}=x-q F_{0}\right.$ and $\left.y_{r}=y-r F_{0}\right)$, and $\Delta \phi(x, y) \equiv \phi\left(x+x_{0} / 2, y\right)-\phi\left(x-x_{0} / 2, y\right)$. The pattern would present a phase shift $\xi(\psi)=2 \psi$ and fringe modulation of unity. This configuration uses the CSI as a beam divider and to produce the shear.

Figure 5 shows the diffraction pattern generated by a phase grid. Fig. 5(a) shows the diffraction orders' overlap, indicated by a subscript. Fig. 5(b) shows the diffraction orders generated by the grating; it can be seen that there are at least 9 diffraction orders with comparable intensities. This result is due to the modulation generated by coefficients $J_{n, l}$. Fig. 5(c) shows the replicas of the interference patterns centered on each diffraction order.

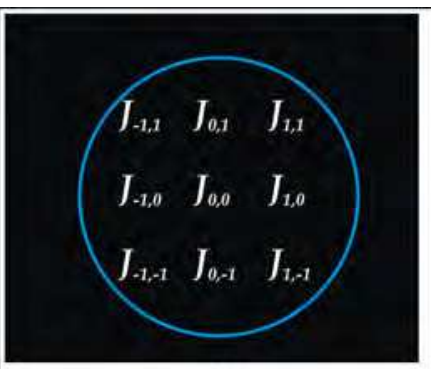

(a)

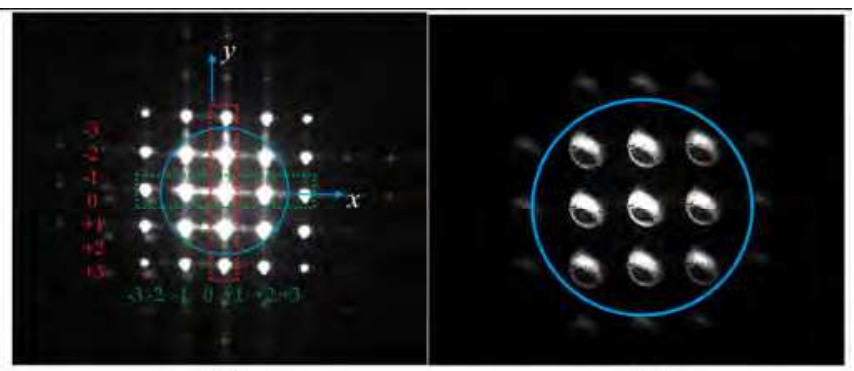

(b) (c)

Fig. 5. Replicas of the interference patterns. (a) Fourier coefficients for each replica of the interference pattern. (b) Experimental diffraction orders generated by high frequency BiRonchi gratings (100 ln/mm). (c) Replicas of the interference patterns centered on each diffraction order (Circle).

\section{Slope measurements of a phase object}

In general, if an object without absorption (transparent) is placed on one arm of the interferometer, it is expressed as: 


$$
O(x, y)=e^{i \cdot \phi(x, y)}
$$

where $\phi(x, y)$ is a real function; this object is known as a phase object. For this analysis, we consider the special case where $|\phi(x, y)|<1$ for both $|\phi(x, y)|^{2}<<1$, resulting in the kind of transparent objects known as phase objects. Accepting the approach

$$
\begin{aligned}
& O(x, y)=e^{i \cdot \phi(x, y)} \\
& O(x, y)=1+i \cdot \phi(x, y)
\end{aligned}
$$

and taking the partial derivative of Eq. (26) with respect to $x$, we obtain

$$
\frac{\partial}{\partial x} O(x, y)=c_{0} \cdot \frac{\partial}{\partial x} \phi(x, y)
$$

with $c_{0}=i$ being a constant. It can be noted that the derivative of the phase object is proportional to the derivative of the phase, while additionally maintaining a constant phase relationship $\pi / 2$ because $i=e^{i \cdot \pi / 2}$. It is well known that when an object is illuminated by a single collimated beam, the relationship between the phase difference and the change in displacement [Rastogi, 1996; Ng , 1995; Bhaduri, 2006] is obtained from:

$$
\frac{\partial \phi(x, y)}{\partial x}=\frac{2 \pi}{\lambda} \cdot\left[\sin \theta \frac{\partial d u}{\partial x}+(1+\cos \theta) \frac{\partial w}{\partial x}\right] \Delta x
$$

where $u$ and $w$ specify the displacement components. The fringe pattern has contributions from the deformation $\partial u / \partial x$ and the slope $\partial w / \partial x$. However, when the object is illuminated in the normal direction $\left(\theta=0^{\circ}\right)$, the fringe pattern represents the $x$-partial slope, $\partial w / \partial x$. This is:

$$
\frac{\partial \phi(x, y)}{\partial x}=\frac{4 \pi}{\lambda} \cdot\left[\frac{\partial w(x, y)}{\partial x}\right] \Delta x
$$

giving us the slope of a phase object as

$$
\frac{\partial w(x, y)}{\partial x}=\frac{c_{1}}{c_{0}} \cdot \frac{\partial}{\partial x} O(x, y)
$$

with $c_{1}=\lambda / 4 \pi \cdot \Delta x$.

For the case of the radial slope, Eq. (29) can be used only with the consideration of the radial dependency.

\subsection{Static distributions}

For the case of measurements of the slope in the $x$-direction, experimental results are presented in Figures 6 and 7 for a microscope slide and an acetate film, respectively. The 
polarizer arrays used for these two proposed cases are presented in section 2.1 (See Fig. 1). In Fig. 6 (Case: configuration I), the upper row shows a microscope slide blocking half of the beam, with an inclination of $5^{\circ}$ to axis $z$, resulting in a modification of the slope of the fringes. The lower row shows the results obtained with a water drop flowing into a microscope slide (since it modifies the surface of slide, the phase introduced by the drop is equivalent to obtaining a deformation in the $\mathrm{z}$ direction, out of plane). Fig. 7 shows the case of Configuration II of the polarizer array, where the microscope slide has been placed with a minimum inclination (above); as a result, the unwrapped phase presents the slope obtained. The second case shows water deposited on a microscope slide presenting variations in the surface. It can be seen that the patterns used with polarizer arrangement $I$ have an apparent decrease of the contrast of the fringes; this is because in both cases, the samples had residual dust particles, and the water was not entirely transparent, so there was minimal absorption of the medium.

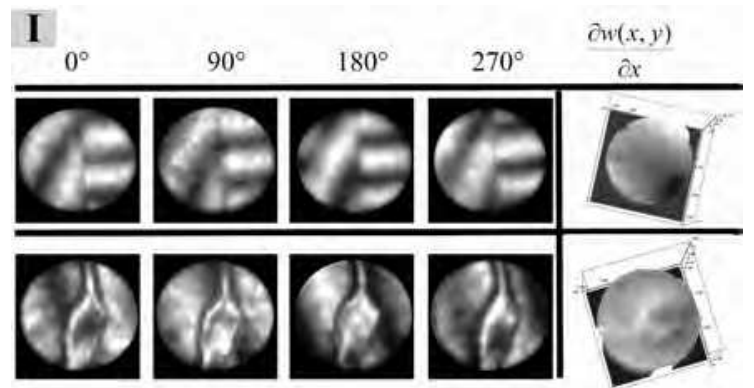

(a) (b)

Fig. 6. Microscope slide (Configuration I of polarizer's array). The relative shifts corresponding to polarizing filter angles in degrees were $\psi_{1}=0 \quad \psi_{2}=46.577^{\circ} \quad \psi_{3}=92.989^{\circ} \quad \psi_{4}=136.42^{\circ}$ (a) Interference patterns obtained in a single shot (b) Retrieved Slope.

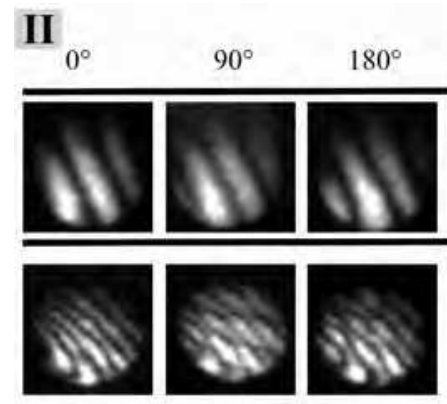

(a)

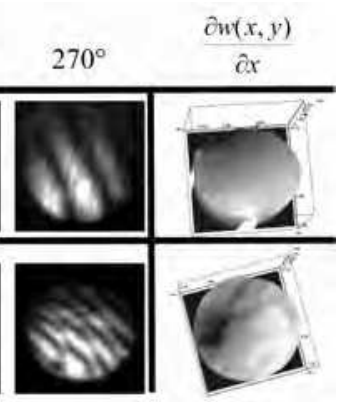

(b)

Fig. 7. Microscope slide (Configuration II of polarizer's array). The relative shifts corresponring to polarizing filter angles in degrees were $\psi_{1}=0 \quad \psi_{2}=46.577^{\circ} \quad \psi_{3}=92.989^{\circ} \quad \psi_{4}=136.42^{\circ}$ (a) Interference patterns obtained in a single shot (b) Retrieved Slope. 
The experimental results for an acetate sample (average thickness of about 100 microns), on which a deformation in the $z$ direction was caused, are shown in Fig. 8, where the patterns obtained in simultaneous capture and the slope associated with the derivative of the phase for configuration I of the polarizer array are presented. In both cases, it can be seen that the fringe pattern changes to generate a deformation around a point on the acetate.

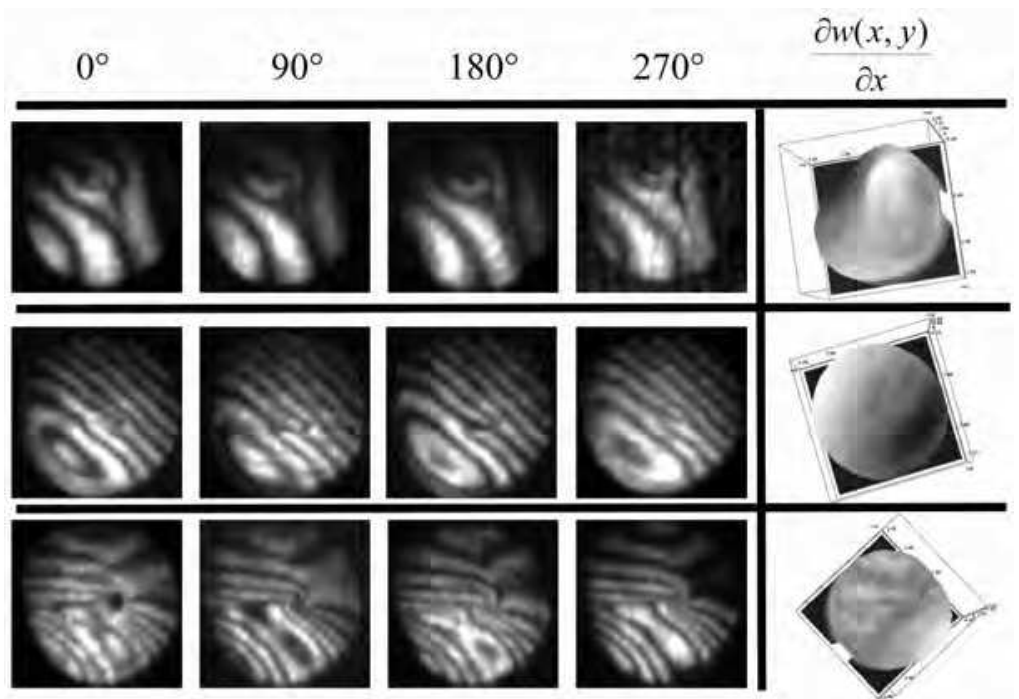

(a) (b)

Fig. 8. Acetate. The relative shifts corresponding to polarizing filter angles in degrees were $\psi_{1}=0 \quad \psi_{2}=46.577^{\circ} \quad \psi_{3}=92.989^{\circ} \quad \psi_{4}=136.42^{\circ}$ (a) Interference patterns obtained in a single shot (b) Retrieved Slope.

Experimental results are presented using the optical system described in section 2.2 (Fig. 2). In order to extract the radial slope of a phase object, it is only analyzed using four patterns obtained in a single shot, and applying the classical method of phase extraction. Fig. 9 shows experimental results corresponding to several samples under study and their associated radial slopes. The upper row in Fig. 9 presents the radial slope of an ophthalmic lens presenting a cross section of $5 \mathrm{~mm}$, the center row shows the deformed interference patterns of one drop of immersion oil placed on a microscope slide, and the lower row presents the deformation caused by lubricating oil over a microscope slide. The fringe pattern shows stress associated with the stabilization on the slide (perpendicular lines in the concentric pattern). This variation can be observed due to the radial slope variation. 


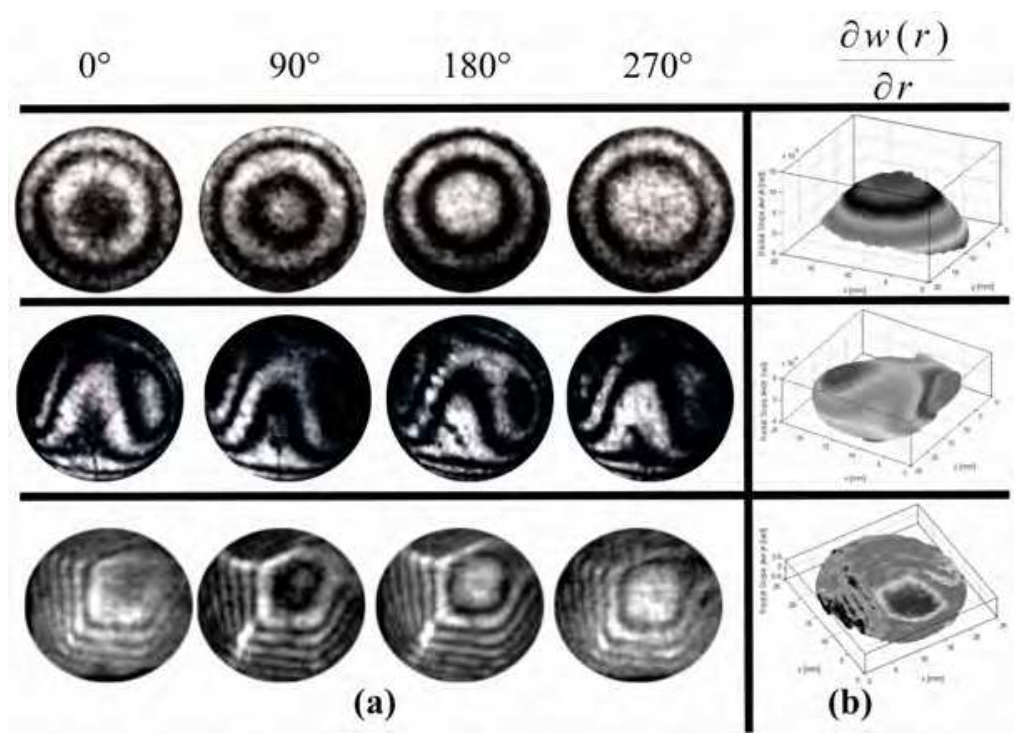

Fig. 9. Radial slope measurements. (a) Interference pattern captured in a single shot and its respective (b) radial slope retrieved.

\subsection{Dynamic distributions}

A dynamic phase object is shown in Fig. 10 where a transparent bubble is crossing the field of the CCD camera. The corresponding interferograms for successive captures are presented. These results show the curvature of the semi-sphere of the phase object producing a characteristic distortion of a spherical wavefront in the primary interference pattern (circle). In the animation, it is possible to see the transition that takes place when the bubble bursts and the interference patterns recover their initial profile (dot circle). The slope was calculated obtaining the phase unwrapping, according to Eq. (27). Before calculating the unwrapped phase, each interferogram was normalized and filtered using a low-pass filter.

For the case of radial symmetry, a dynamic event (4-D) is presented. Evaporation of lubricating oil placed on a microscope slide by using a tin soldiering iron is presented in Figure 11. It shows the change in the interference pattern (upper row) and its associated radial slope (lower row); the radial slope transition that occurs when the oil is evaporated can be seen. It is important to show that the results are obtained using an optical table without pneumatic suspension, and that the polarized array used is formed by conventional polarized film placed at different angles, having the advantage of not using a micropolarized array. 


\section{Event in 4-D}
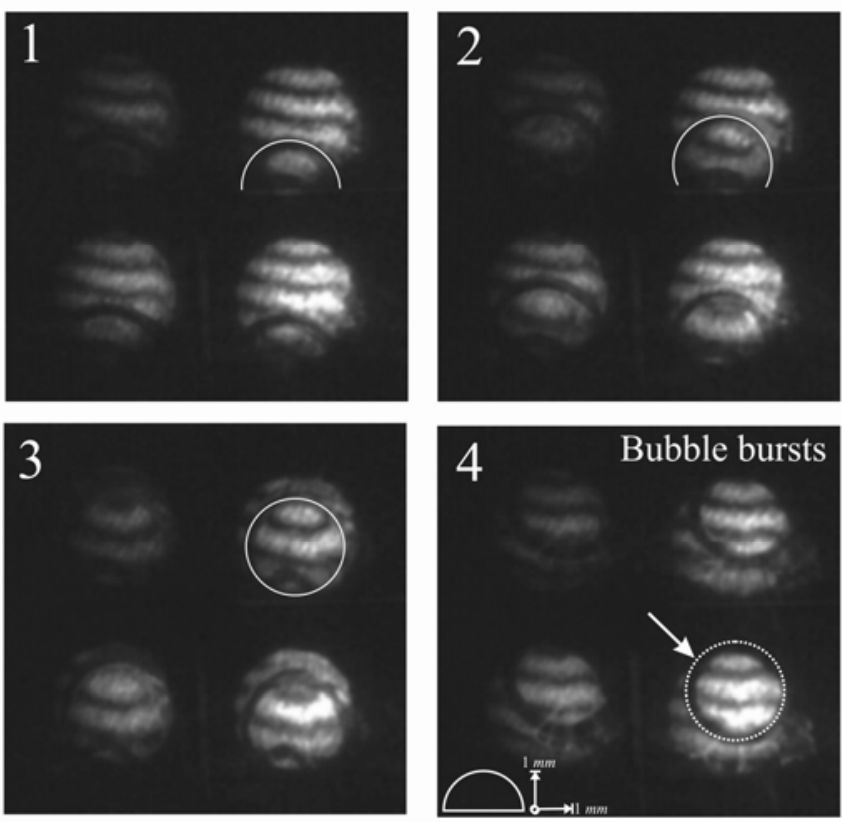

Fig. 10. Dynamic phase object. Transparent bubble moving on a microscope slide. Representative Frames.

\section{Event in 4-D}

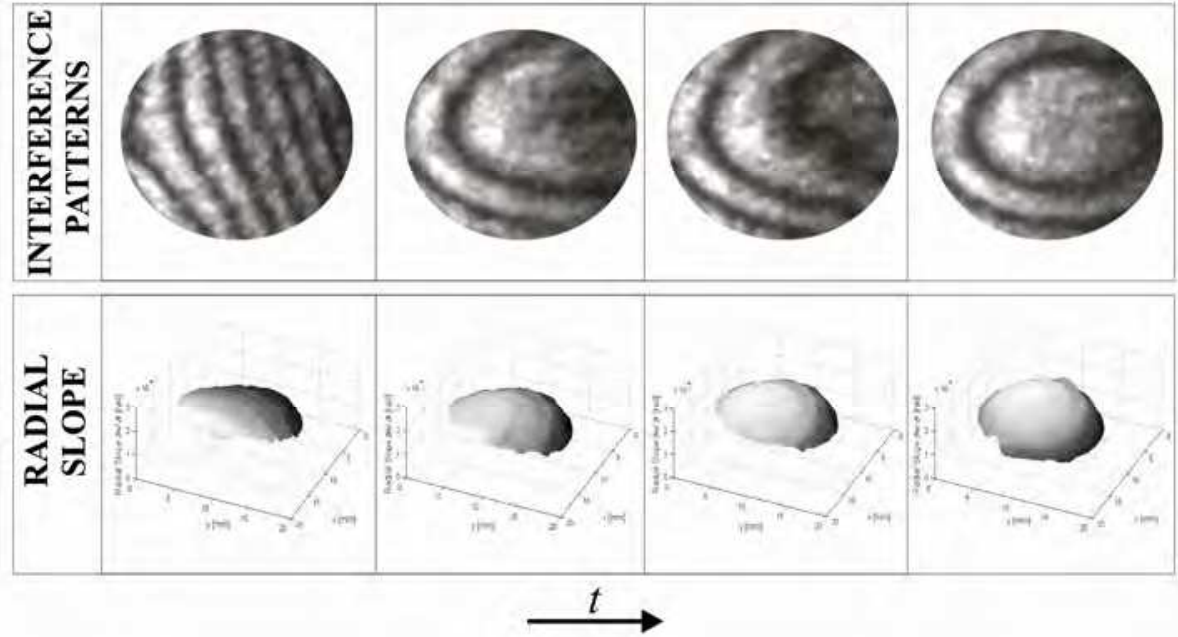

Fig. 11. Dynamic distributions of the radial slope are presented in 4-D. Representative Frames. Upper Row: Interference patters. Lower row: Evolution of the radial slope. 


\section{Conclusion}

With the presented interferometric systems, the slope of phase objects can be obtained in a single shot in real time by using the diffraction properties of high frequency Ronchi gratings. It is suitable to replace the Ronchi gratings used in other systems by phase gratings when it is necessary to implement systems capable of obtaining $N$-interferograms. It should be noted that the elements used are inexpensive and easily accessible; as a result, the system presented can be easily implemented for various applications in single shot polarizing phase shifting interferometry.

For the case of the radial derivative, an adaptation of the MZRI with a phase grid to simultaneously capture several shearograms for slope measurements through the use of phase-shifting techniques has been demonstrated. We present different experimental results obtained by a radial shear interferometer capable of studying events.

The advantage of using these systems is that the lateral or radial slope of the phase object is obtained in real time, allowing us to study transparent samples with this symmetry or dynamic events of transparent samples, which requires obtaining real-time directional derivatives. As future work, we intend to use these systems as wave front sensors, for temperature measurements, or in optical tomography applications.

\section{Acknowledgments}

Authors thank M. A. Ruiz for his contribution in proofreading the manuscript. Partial support from "Consejo Nacional de Ciencia y Tecnología (CONACYT)" and "Centro de Investigaciones en Óptica A.C. (CIO)" through projects 290597 (CONACYT-CIO) and 124145 (CONACYT-BUAP) is also acknowledged. Author N. I. Toto-Arellano expresses sincere appreciation to Lichita, Miguelon and Damian-El, for the support provided, and to CONACYT for grant 102137/43055. Author D. I. Serrano-García (Grant:227470/31458) is very grateful to CONACyT for the graduate scholarship granted, and expresses sincere appreciation to Geliztle.

\section{References}

Barrientos-García B., Moore A. J., Pérez-López C., Wang L., and Tschudi T.,(1999). Transient deformation measurement with electronic speckle pattern interferometry by use of a holographic optical element for spatial phase stepping, Appl. Opt., Vol. 38, pp. 5944-5947 (1999).

Bhaduri B., Krishna Mohan N., and Kothiyal M. P., (2006). A dual function ESPI system for the measurement of out-of-plane displacement and slope," Opt. Lasers Eng. Vol. 44, pp. 637-644.

Cheng Y.-Y and Wyant J. C., (1984). Two-wavelength phase shifting interferometry, Appl. Opt., Vol. 23, pp. 4539-4543.

Chen L. C., Yeh S. L., Tapilouw a. M. and Chang J. C., (2010). 3-D surface profilometry using simultaneous phase-shifting interferometry, Opt. Commun., Vol. 283, pp. 3376- 3382.

Cordero-Dávila A., Luna-Aguilar E., Vázquez-Montiel S., Zárate-Vázquez S. and PercinoZacarías M. E. (1998). Ronchi test with a square grid, Appl. Opt., Vol. 37, pp. 672675. 
Geary J. M., R. K. Tyson ed., (2000).Wavefront sensors," C.IV in Adaptive Optics Engineering Handbook pp. 123-150.

Goodman, Jooseph W.; J. Wiley \& Sons (1988). Introduction to Fourier optics.

Hariharan P, Oreb B. F., Wanzhi Z.,(1984). Measurement of aspheric surfaces using a microcomputer controlled digital radial-shear interferometer, Jour. Mod. Optics, Vol. 31, pp. 989-999.

Hernandez-Gomez C, Collier J. L. , Hawkes S. J., Danson C. N., Edwards C. B., Pepler D. A., Ross I. N., Winstone T. B. (2000). Wave-front control of a large-aperture laser system by use of a static phase corrector, Appl. Opt. Vol. 39, pp. 1954-1961.

Hutchin R. A.,(1985). Combined shearing interferometer and Hartmann wavefront sensor, Pat. Num. 4518854.

Kowalik W. W., Garncarz B. E., Kasprzak H. T., (2002). Corneal topography measurement by means of radial shearing interference: part I- theoretical considerations, Optik 113 , pp. 39-45.

Lago E. L. and Fuente R. ,(2008). Amplitude and phase reconstruction by radial shearing interferometry, Appl. Opt., Vol. 47, pp. 372-376.

Liu D., Yang Y., Wang L., Zhuo Y., (2007). Real-time diagnosis of transient pulse laser with high repetition by radial shearing interferometer, Appl. Opt., Vol. 46, pp. 83058314.

Malacara D., (1974). Mathematical interpretation of radial shearing interferometers, Appl. Opt., Vol. 13, pp. 1781-1784.

Malacara D., Servin M., and Malacara Z.; Marcel Dekker (1998). Interferogram Analysis for Optical Testing.

Meneses-Fabian C., Rodriguez-Zurita G., and Arrizón V., (2006). Optical tomography of transparent objects with phase-shifting interferometry and stepwise-shifted Ronchi ruling, J. Opt. Soc. Am. A, Vol. 23, pp. 298-305.

Mercer C. R. and Creath K.,(1996). Liquid-crystal point-diffraction interferometer for wavefront measurements, Applied Optics, Vol. 35, pp. 1633.

Neal M.J., Ph.D. Thesis, University of Arizona (2003). Polarization Phase-Shifting PointDiffraction Interferometer.

$\mathrm{Ng}$ T. W., (1995). Digital speckle pattern interferometer for combined measurements of outof-plane displacement and slope, Opt. Commun., Vol. 116, pp. 31-35.

Nomura T., Murata S., Nitanai E., and Numata T, (2006). Phase-shifting digital holography with a phase difference between orthogonal polarizations, Appl. Opt., Vol. 45, pp. 4873-4877.

Novak M., Millerd J., Brock N., North-Morris M., Hayes J. and Wyant J.C., (2005). Analysis of a micropolarizer array-based simultaneous phase-shifting interferometer, Appl. Opt., Vol. 44, pp. 6861-6868.

Rastogi P. K., (1996). Measurement of in-plane strains using electronic speckle and electronic speckle- shearing pattern interferometry, J. Mod. Opt., Vol. 43, pp. 1577-1581.

Ronchi V.,(1964). Forty Years of History of a Grating Interferometer, Appl. Opt., Vol. 3, pp. 437-451.

Rodriguez-Zurita G., Toto-Arellano N. I., Meneses-Fabian C. and Vázquez-Castillo J. F.,(2008).One-shot phase-shifting interferometryfive, seven, and nine interferograms, Opt Letters, Vol. 33, pp. 2788-2790. 
Rodríguez-Zurita G., Toto-Arellano N. I., Meneses-Fabian C. and Vázquez-Castillo J. F., (2009). Adjustable lateral-shear single-shot phase-shifting interferometry for moving phase distributions, Meas. Sci. Technol. Vol. 20, pp. 115902.

Sharma D. K., Sirohi R. S. and Kothiyal M. P., (1984). Simultaneous measurement of slope and curvature with a three-aperture speckle shearing interferometer, Appl. Opt., Vol. 23, pp. 1542-1546.

Serrano-García D.I., Toto-Arellano N.I., Martínez García A., Rayas Álvarez J.A., TéllezQuiñones A., and Rodríguez-Zurita G.,(2011). Simultaneous phase-shifting cyclic interferometer for generation of lateral and radial shear, Rev. Mex. Fís., Vol. 57, pp. 255-258.

Shirai T. H. Barnes T. G. Haskell (2001),Real-time restoration of a blurred image with a liquid-crystal adaptive optics system based on all-optical feedback interferometry, Opt. Commun., Vol. 118, pp. 275-282.

Steel W. H., (1965). A radial shear interferometer for testing microscope objectives, J. Sci. Instrum., Vol. 42, pp. 102-104.

Toto-Arellano N. I., Rodríguez-Zurita G., Meneses-Fabian C., Vazquez-Castillo J. F., (2008). Phase shifts in the Fourier spectra of phase gratings and phase grids: an application for one shot phase-shifting interferometry, Opt. Express, Vol. 16, pp. 19330- 19341

Toto-Arellano N.I., Rodriguez-Zurita G., Meneses-Fabian C. and Vázquez-Castillo J.F., (2009).A single-shot phase-shifting radial-shearing interferometer, J. Opt. A: Pure Appl. Opt., Vol. 11, pp, 045704.

Toto-Arellano N. I., Martínez-García A., Rodríguez-Zurita G., Rayas-Á lvarez J.A., and Montes-Perez A., (2010). Slope measurement of a phase object using a polarizing phase-shifting high-frequency Ronchi grating interferometer, Appl. Opt., Vol. 49, pp. 6402-6408.

Wyant J.C. (2004). Vibration insensitive interferometric optical testing, in Frontiers in Optics, OSA Technical Digest, OTuB2.

Yamaguchi I. and Zhang T., (1997). Phase-shifting digital holography, Opt. Lett., Vol. 22, pp. 1268-1270. 


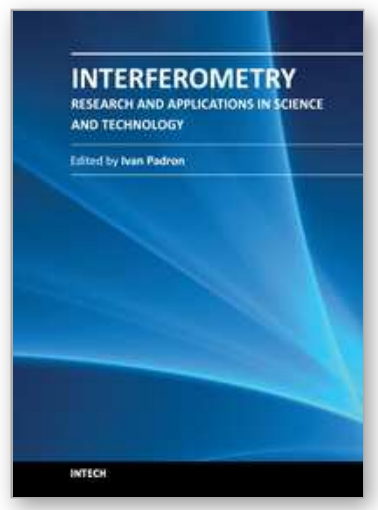

\section{Interferometry - Research and Applications in Science and Technology}

Edited by Dr Ivan Padron

ISBN 978-953-51-0403-2

Hard cover, 462 pages

Publisher InTech

Published online 21, March, 2012

Published in print edition March, 2012

This book provides the most recent studies on interferometry and its applications in science and technology. It is an outline of theoretical and experimental aspects of interferometry and their applications. The book is divided in two sections. The first one is an overview of different interferometry techniques and their general applications, while the second section is devoted to more specific interferometry applications comprising from interferometry for magnetic fusion plasmas to interferometry in wireless networks. The book is an excellent reference of current interferometry applications in science and technology. It offers the opportunity to increase our knowledge about interferometry and encourage researchers in development of new applications.

\section{How to reference}

In order to correctly reference this scholarly work, feel free to copy and paste the following:

Noel-Ivan Toto-Arellano, David-Ignacio Serrano-García, Amalia Martínez-García and Gustavo RodríguezZurita (2012). Simultaneous Phase Shifting Shearing Interferometry for Measurement of Static and Dynamic Phase Objects, Interferometry - Research and Applications in Science and Technology, Dr Ivan Padron (Ed.), ISBN: 978-953-51-0403-2, InTech, Available from: http://www.intechopen.com/books/interferometry-researchand-applications-in-science-and-technology/simultaneous-phase-shifting-shearing-interferometry-formeasurement-of-static-and-dynamic-phase-obje

\section{INTECH}

open science | open minds

\author{
InTech Europe \\ University Campus STeP Ri \\ Slavka Krautzeka 83/A \\ 51000 Rijeka, Croatia \\ Phone: +385 (51) 770447 \\ Fax: +385 (51) 686166 \\ www.intechopen.com
}

\author{
InTech China \\ Unit 405, Office Block, Hotel Equatorial Shanghai \\ No.65, Yan An Road (West), Shanghai, 200040, China \\ 中国上海市延安西路65号上海国际贵都大饭店办公楼 405 单元 \\ Phone: +86-21-62489820 \\ Fax: +86-21-62489821
}


(C) 2012 The Author(s). Licensee IntechOpen. This is an open access article distributed under the terms of the Creative Commons Attribution 3.0 License, which permits unrestricted use, distribution, and reproduction in any medium, provided the original work is properly cited. 